\title{
Morfopatología Proventricular en Patos Silvestres (Anas discors) Infestados con Tetrameresamericana
}

\author{
Proventricular Morphopathology in Wild Ducks (Anas discors) Infested \\ wITH Tetrameres americana
}

\author{
Manuel Colas C. ${ }^{1,4}$, Raiden Grandía G. ${ }^{2,5}$, Daisy Rodríguez G. ${ }^{3}$, Jorge Demedio L. ${ }^{1}$, \\ Irma Menéndez B. ${ }^{1}$, Efrén García T. ${ }^{1}$
}

\section{Resumen}

\begin{abstract}
El estudio tuvo como objetivo describir los cambios morfopatológicos en proventrículo de patos silvestres (Anas discors) infestados con Tetrameres americana. Se realizó la necropsia a 89 cadáveres de patos adultos procedentes de Pinar del Río, Cuba, y se tomó un fragmento de $1 \mathrm{~cm}^{2}$ de 22 proventrículos con diagnóstico clínico de proventriculitis parasitaria para el estudio histopatológico. Los órganos se colectaron para su análisis parasitológico, clasificando las proventriculitis en leve y severa, según la cantidad de glándulas comprometidas con el nematodo y la magnitud del exudado catarral. El 25\% (22/89) de las aves presentó proventriculitis catarral con presencia de T. americana, donde el $73 \%$ de los casos fue considerado como leve. Se observó erosión de la mucosa, degeneración con necrosis y formación de nódulos de $1 \mathrm{~cm}$ de diámetro en la yema glandular. Se concluye que la infestación con $T$. americana causa daños morfopatológicos en el proventrículo de A. discors.
\end{abstract}

Palabras clave: morfopatología proventricular, Tetrameres americana, Anas discors

\section{Abstract}

The aim of this study was to describe the morphopathological changes in proventriculus of wild ducks (Anas discors) infested with Tetrameres americana. Necropsy to 89 cadavers of adult ducks from Pinar del Río, Cuba, was performed. Samples of $1 \mathrm{~cm}^{2}$

\footnotetext{
${ }^{1}$ Facultad de Medicina Veterinaria, Universidad Agraria de La Habana, UNAH, Cuba

${ }^{2}$ Dirección de Biotecnología, Centro Nacional para la Producción de Animales de Laboratorio, CENPALAB, Cuba

${ }^{3}$ Laboratorio de Investigación y Diagnóstico Aviar «Jesús Menéndez», LIDA, Cuba

${ }^{4}$ E-mail: manuelcc@unah.edu.cu

${ }^{5}$ E-mail: raiden.grandia@cenpalab.cu
}

Recibido: 19 de octubre de 2015

Aceptado para publicación: 9 de marzo de 2016 
from 22 proventriculi diagnosed with parasitic proventriculitis were taken for the histopathological study. All proventriculi ducts were collected for parasitological analysis and the lesions were classified as mild and severe according to the amount of glands affected with the nematode and the magnitude of catarrhal exudate. The results showed that $25 \%(22 / 89)$ of the ducks had proventriculitis caused by $T$. americana where $73 \%$ of the cases was considered as mild. Mucosal erosion, degeneration with necrosis and formation of nodules of $1 \mathrm{~cm}$ of diameter in the glandular yolk were observed. It is concluded that infestation with $T$. americana causes morphopathological lesions in proventriculus of $A$. discors.

Key words: proventricular morphopathology, Tetrameres americana, Anas discors

\section{INTRODUCCIÓN}

Las aves migratorias pueden diseminar una amplia gama de agentes patógenos así como ser portadoras aparentemente sanas de enfermedades aviares (Hubálek, 2004). Dada su capacidad de volar libremente y cubrir largas distancias durante las migraciones anuales, estas aves juegan un papel potencial en la epidemiología de diversas zoonosis (Abulreesh et al., 2007).

En Cuba, el 90\% de los patos cazados están representados por patos de la Florida (A. discors) (Fernández, 1968; Acosta et al., 2003; Garvon et al., 2011). Es un ave anseriforme migratoria de la Familia Anatidae y nativa del continente americano. Estas aves migran desde Alaska hacia el sur de EEUU, por ambas costas, llegando a América Central y Suramérica (Chile y Argentina). Asimismo, son visitantes habituales en invierno de las islas del Caribe (Todd, 1996).

El tracto digestivo de las aves de la Familia Anatidae puede ser infestado por helmintos, en particular el estómago glandular. Entre estos parásitos se destacan especies del género Tetrameres (T. americana, T. ryjikovi, T. striata) y de la Familia Spiruridae (= Tetrameridae) que ocasionan la espirurosis o tetramerosis en aves (Sánchez y Lamazares, 2010).
T. americana tiene dimorfismo sexual. Los machos son blancos y delgados con los extremos afilados y miden $5.3 \mathrm{~mm}$ de largo, mientras que las hembras son globulosas y rojas, de $4 \mathrm{~mm}$ de diámetro (Biu y Haddabi, 2005). Tiene como hospederos intermediarios a Blattella germanica, Melanoplus differentialis, M. femurrubrum, Conocephalus saltator, Scyllina cyanipes, Dendrophilus sp, Dermestes vulpinus, Epitragus diremptus, Gonocephalus seriatum, Euborellia annulipes y Orchestia platensis. Este nematodo tiene gran afinidad por las glándulas del proventrículo de diversos grupos de aves silvestres y acuáticas como Anseriformes, Podicipediformes, Ciconiiformes, Falconiformes, Galliformes, Gruiformes, Charadriiformes, Columbiformes y Strigiformes (hospederos definitivos) (McDonald, 1969; Anderson, 2000; Villarreal et al., 2011).

Zocche y Silva (2009) indican que $T$. americana tiene afinidad por las criptas glandulares del proventrículo. Dentro de los cambios morfopatológicos que ocasiona T. americana, Everett y Beltsville (1968) reportaron en gallinas la presencia de exudación catarral, engrosamiento y exfoliación de las paredes del proventrículo, así como necrosis glandular extensa con pérdida total de la mucosa. Otros estudios demostraron lesiones en la pared del proventrículo de palomas (Columba livia) (González et al., 2004), 
guacamayos (Ara ararauna) (Silva et al., 2005) y pollos (Kamani et al., 2010), demostrando una gran adaptabilidad a diversas especies de aves.

Estudios de prevalencia de T. america$n a$ señalan una frecuencia de $39 \%$ en pollos de traspatio en Nigeria (Biu y Haddabi, 2005), $52 \%$ en Nicaragua (Luna-Olivares et al., 2006), 57\% en Zimbabwe (Percy et al., 2012) y 67\% en Sudáfrica (Mukaratirwa y Khumalo, 2012). Es así que el presente estudio tuvo como objetivo demostrar los cambios morfopatológicos en proventrículo de patos silvestres (A. discors) infestados con T. americana.

\section{Materiales y Métodos}

En el estudio se trabajó con 89 cadáveres de patos silvestres (A. discors) adultos provenientes del embalse «Los Palacios», de la provincia Pinar del Río, Cuba. Las aves fueron cazadas por los autores del estudio con previa autorización de la Federación Cubana de Caza Deportiva, transportadas y conservadas en un contenedor refrigerado (2$8^{\circ} \mathrm{C}$ ) entre 2 y 3 horas antes de su llegada al laboratorio. La necropsia se realizó en las áreas de anatomía patológica y de parasitología del Laboratorio de Investigación y Diagnóstico Aviar «Jesús Menéndez» (LIDA), en La Habana, Cuba.

En el laboratorio se identificaron posibles lesiones en piel. La necropsia se realizó siguiendo los lineamientos de Sánchez y Lamazares (2010). Se retiró parte de la piel y se hizo una incisión longitudinal desde la base del pico hasta la cloaca para la exposición de todo el aparato digestivo (Friend et al., 1999; Martín et al., 2004). Se tomaron muestras de $1 \mathrm{~cm}^{2}$ de proventrículos con diagnóstico de proventriculitis parasitaria $(n=22)$, las cuales se fijaron en formalina tamponada al $10 \%$, se incluyeron en parafina y se hicieron cortes de $4 \mu \mathrm{m}$ que fueron teñidos con Hematoxilina-Eosina para el análisis histopatológico.
El resto de los conductos proventriculares se colectaron en placas petri estériles y se almacenaron a $4{ }^{\circ} \mathrm{C}$ para la identificación de la familia y especie helmíntica, según los criterios de Rodríguez et al. (2003) y Morishita y Schaul (2007). Se aplicaron los métodos helmintológicos directo y de flotación (solución sobresaturada de $\mathrm{NaCl}$ ) para la observación de machos adultos y huevos embrionados de T. americana en las heces, con la ayuda de la tinción con lugol y un microscopio óptico (Opton) de luz ordinaria a 400X. Para la identificación de las hembras adultas se realizaron incisiones sobre las glándulas infestadas y se colectaron con pinza en placas petri con agua destilada para su observación bajo estereoscopio (Olympus).

Se realizó un análisis semicuantitativo de las proventriculitis parasitarias identificadas mediante la cuantificación de glándulas con evidencia parasitaria, así como la severidad en cada proventrículo inflamado, considerándose como Leve (+) hasta 10 glándulas con evidencia parasitaria y exudación catarral ligera, y Severa (++) la presencia de más de 10 glándulas con evidencia parasitaria e hipersecreción de mucus.

En el análisis estadístico se compararon las proporciones de ausencia y presencia de proventriculitis parasitaria causada por $T$. americana, así como entre los grados de proventriculitis. Se consideró una $\mathrm{p}<0.05$ como significación estadística, utilizando el programa estadístico COMPAPROP v. 2.1.

\section{Resultados y Discusión}

En el análisis macroscópico se evidenció que el 25\% (22/89) de los patos $(A$. discors) presentaron proventriculitis parasitaria causada por abundantes hembras adultas de T. americana, hallazgo que no había sido notificado en Cuba. Asimismo, se observaron en el microscopio abundantes huevos embrionados de $T$. americana en todas las muestras de heces de las aves afectadas pero 


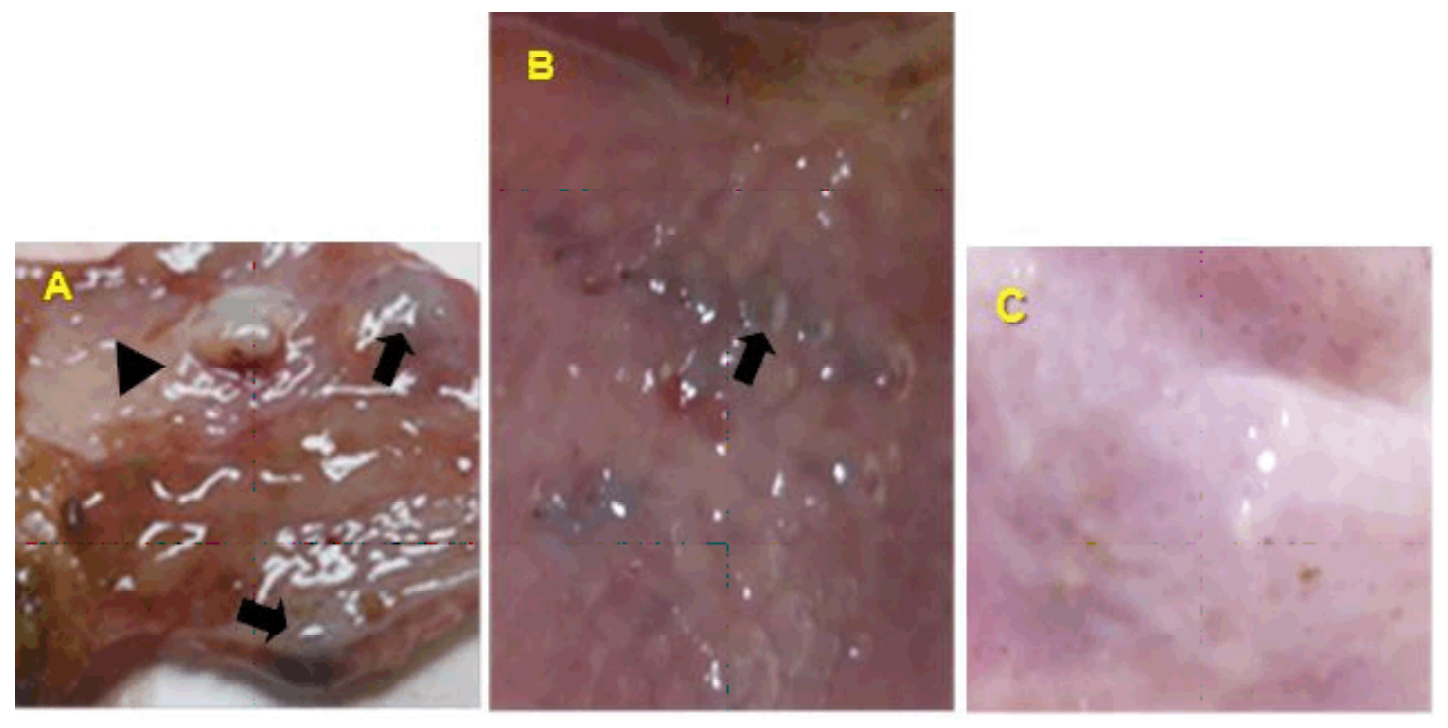

Figura 1. Proventriculitis parasitaria en patos silvestres (A. discors) infestados con Tetrameres americana. A: Exudado catarral de la mucosa (cabeza de flecha) y glándulas con elevaciones prominentes (nódulos de $1 \mathrm{~cm}$ de diámetro) y evidencia parasitaria en su interior (flecha). B: Mucosa glandular con abundantes nematodos (flecha). C: Mucosa glandular sin alteración aparente

con escasos machos adultos, lo que infiere un periodo posinfestación superior a los 45 días (Morishita y Schaul, 2007).

Existen diversos reportes de infestación en aves por varias especies del género Tetrameres. En este sentido, Méndez et al. (1998) encontraron siete palomas infestadas con T. confusus de 11 parasitadas, Bergan et al. (1994) reportaron infestaciones de $T$. striata en varias especies de patos ( $A$. platyrhynchos, A. falcata, A. acuta, A. discors) y Al-Moussawi (2014) notificó a $T$. americana en proventrículo y estómago muscular de patos cucharetas (Anas clypeata). Asimismo, Kamil et al. (2011) hallaron a T. fissispina en patos con severas lesiones en tráquea, pulmón, corazón, hígado, páncreas, bazo, riñón, serosa intestinal y mesenterio, así como formación de numerosos nódulos en la pared proventricular. Estos hallazgos hacen evidente la gran afinidad de estos nematodos por el estómago glandular de las aves, en especial de patos silvestres.
E1 $73 \%$ de los patos con proventriculitis parasitaria presentó lesiones leves, en tanto que el restante $27 \%$ presentó lesiones severas. En los proventrículos se observaron yemas gástricas prominentes que comprometían la submucosa, así como la presencia de hipersecreción de mucus en la superficie epitelial (Figura 1A). La formación nodular de $1 \mathrm{~cm}$ de diámetro que se muestra en la figura fue muy similar a la observada por Kamil et al. (2011). Asimismo, la arquitectura orgánica de los proventrículos afectados es muy diferente de lo que se puede observar en proventrículos sanos (Figura 1C).

Se observó, asimismo, una marcada infestación parasitaria en el interior de la mucosa glandular (Figura 1B), alteración similar a la encontrada por Colas et al. (2010) en una proventriculitis erosiva-ulcerativa causada por Dispharynx nasuta, con pérdida de las yemas gástricas que involucraban la submucosa, así como pequeños focos de hemorragia en toda la mucosa. 


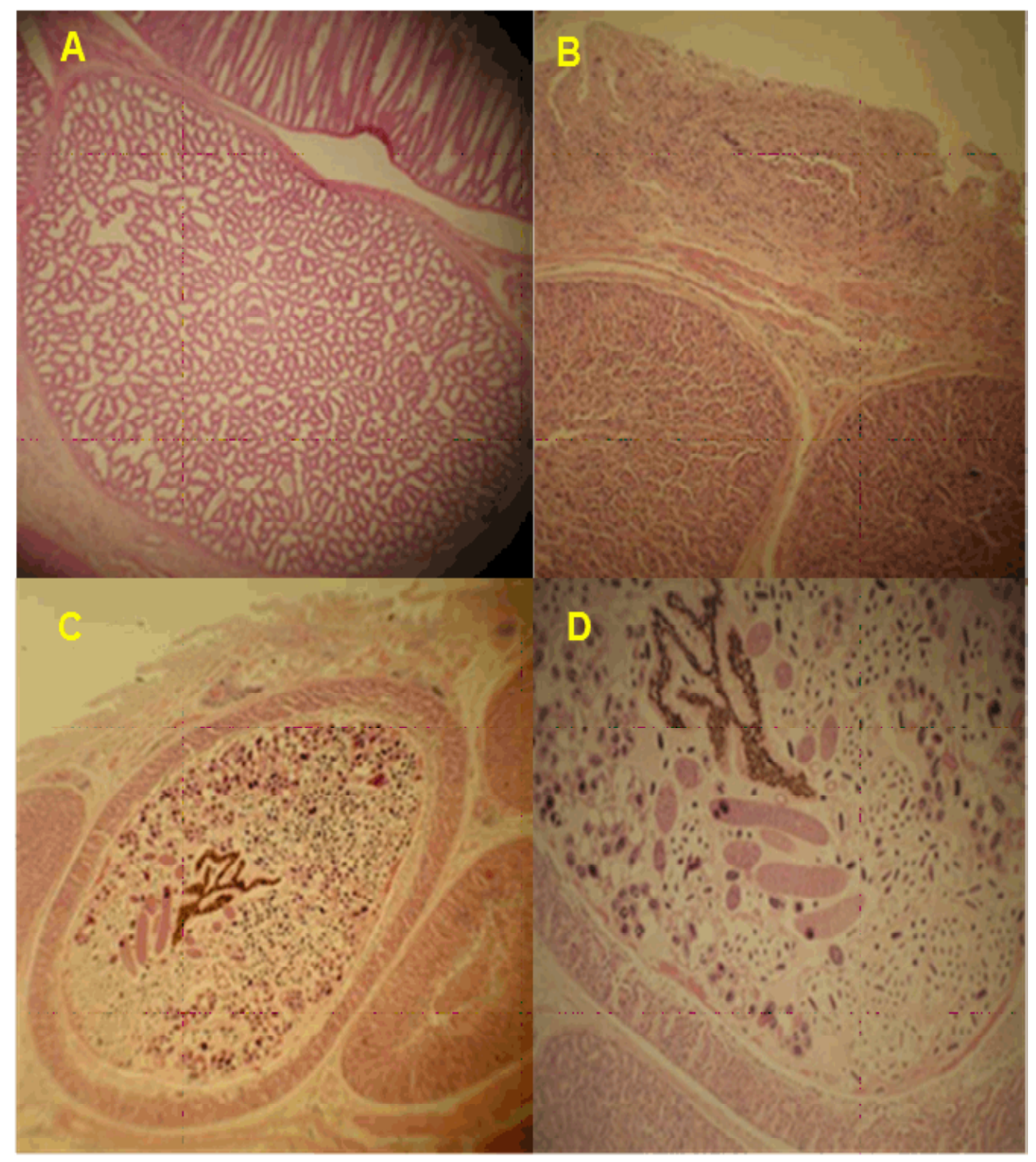

Figura 2. Microfotografía del proventrículo en patos silvestres (A. discors) infestados con Tetrameres americana. A: Mucosa y porción de la submucosa sin alteración aparente. H-E (100X). B: Erosión del epitelio de la mucosa con proliferación de tejido conectivo y degeneración de la yema glandular. H-E (100X). C y D: Degeneración y necrosis del epitelio de la yema glandular con presencia de parte del nematodo rodeado de macrófagos. H-E (200 y 400X, respectivamente)

En el examen histopatológico se observaron erosiones en la mucosa con proliferación de tejido conectivo e intensa eosinofilia, así como degeneración de las células epiteliales, detritus celular epitelial y del parásito (Figuras 2B, 2C). En las glándulas de la yema gástrica se evidenció una intensa pérdida del epitelio simple (Figuras 2B, 2C) con abundantes eosinófilos, células plasmáticas y macrófagos alrededor de las formas parasitarias (Figuras 2C, 2D); alteraciones que coinciden con el reporte de Nagy et al. (1978). 
Estos resultados histopatológicos son similares a los obtenidos por Kamil et al. (2011) en proventrículos de patos infestados con T. fissispina donde hubo dilatación del lumen de la glándula, atrofia, degeneración y descamación de las células glandulares. Asimismo, Bergan et al. (1994) reportaron lesiones nodulares con necrosis, atrofia y descamación del epitelio glandular con infiltración de eosinófilos, linfocitos y macrófagos en $A$. platyrhynchos. Sin embargo, no se observaron cambios morfopatológicos en el resto de los órganos analizados durante la necropsia.

Los hallazgos obtenidos permitieron concluir que la infestación con T. americana causa daños morfopatológicos en el proventrículo de A. discors.

\section{Literatura Citada}

1. Abulreesh HH, Goulder R, Scott GW. 2007. Wild birds and human pathogens in the context of ringing and migration. Ringing Migration 23: 193-200. doi: 10.1080/03078698.2007.9674363

2. Acosta M, Mugica L, Denis D, Rodríguez A, Jiménez A. 2003. Aves comunes en los humedales de Cuba. La Habana, Cuba: Universidad de La Habana. $52 \mathrm{p}$.

3. Al-Moussawi AA. 2014. Stomach nematodes of the shoveler Anas clypeata Linnaeus, 1758 (Anseriformes: Anatidae) wintering in Iraq. Bull Iraq Nat Hist Mus 13: 27-34.

4. Anderson RC. 2000. Order SpiruridaSuborder Spirurina. In: Nematode parasites of vertebrates: their development and transmission. $2^{\text {nd }}$ ed. UK: CABI Publishing. p 383-539.

5. Bergan JF, Radomski AA, Pence DB, Rhodes OE. 1994. Tetrameres (Petrowimeres) striata in ducks. J Wildl Dis 30: 351-358. doi: 10.7589/0090-355830.3.351
6. Biu AA, Haddabi I. 2005. An investigation of Tetrameres infection among local chickens in Maiduguri. Nigerian Vet J 26(1): 44-46.

7. Colas M, Larramendy $R$, Merino A. 2010. Evidencias histopatológicas en un gallo fino cubano infestado por Dispharynx nasuta. Rev Salud Anim 32: 128-131.

8. Everett EW, Beltsville M. 1968. Nemátodos y acantocéfalos de las aves. En: Biester HE, Schwarte LH (eds). Enfermedades de las aves. La Habana: Ed Revolucionaria. p 750-787.

9. Fernández DA. 1968. Aves de caza de Cuba. La Habana: Ed Deportivas. p 4647.

10. Friend M, Franson JC, Ciganovich EA. 1999. Field manual of wildlife diseases: general field procedures and diseases of birds. Madison, USA. US Department of the Interior. $426 \mathrm{p}$.

11. Garvon JM, Fedynich AM, Peterson MJ, Pence DB. 2011. Helminth community dynamics in populations of blue-winged teal (Anas discors) using two distinct migratory corridors. J Parasitol Res 2011 (Art 306257). doi: 10.1155/2011/306257

12. González D, Castillo G, López J, Moreno L, Donoso S, Skewes $O$, Martínez $R$, et al. 2004. Parásitos gastrointestinales y externos de la paloma doméstica (Columba livia) en la ciudad de Chillán, Chile. Agro-Ciencia 20: 107-112.

13. Hubálek Z. 2004. An annotated checklist of pathogenic microorganisms associated with migratory birds. J Wildl Dis 40: 639-659.

14. Kamani J, Meseko CA, Oladokun AT, Tafarki AE, Abba I, Dogo GI. 2010. Tetrameres infection in local poultry in Katsina State, Nigeria. Vet World 3: 130132.

15. Kamil SA, Darzi MM, Mir MS, Shah SA, Shah SN, Khan FA. 2011. Tetrameres fissispina infection in ducks from Bandipora area of Kashmir valley. Israel J Vet Med 66: 43-47. 
16. Luna-Olivares L, Kyvsgaard $N$, Rimbaud E, Pineda N. 2006. Prevalencia y carga parasitaria de helmintos gastrointestinales en gallinas de traspatio (Gallus gallus domesticus), en el municipio de El Sauce, departamento de León, Nicaragua. REDVET 7(11). [Internet]. Disponible en: http:// www.veterinaria.org/revistas/redvet/ n111106/110617.pdf

17. Martín RO, Marín PG, González JS. 2004. Atlas de anatomía de los animales exóticos. España: Masson. 166 p.

18. McDonald EM. 1969. Catalogue of helminths of waterfowl (Anatidae). Bureau of Sport Fisheries and Wildlife. Special Scientific Report Wildlife 126. Washington, USA. 692 p.

19. Méndez V, García NW, Soto M. 1998. Proventriculitis parasitarias por Dispharynx nasuta y Tropisurus confusus en palomas (Columbia livia domestica). Rev Cub Cienc Avícola 22(1):49-51.

20. Morishita TY, Schaul JC. 2007. Parasites of birds. $2^{\text {nd }}$ ed. In: Baker DG (ed). Flynn's parasites of laboratory animals. USA: Blackwell Publishing. $p$ 217-301.

21. Mukaratirwa S, Khumalo MP. 2012. Prevalence of helminth parasites in freerange chickens from selected rural communities in KwaZulu-Natal province of South Africa. J S Afr Vet Assoc 81: 97-101.

22. Nagy C, Birova V, Ovies D. 1978. Alteraciones patológicas en aves afectadas de tropisurosis. Rev Cub Cienc Avícola $9(2)$ : 147-153.

23. Percy J, Pias M, Enetia BD, Lucia T. 2012. Seasonality of parasitism in free- range chickens from a selected ward of a rural district in Zimbabwe. Afr J Agric Res 7: 3626-3631. doi: 10.5897// AJAR12.039

24. Rodríguez DJ, Olivares OJ, Cortez $S$, Larramendy $R$, Zsczypell B, Gómez E, Blandino T, et al. 2003. Métodos para el trabajo con los helmintos más importantes en medicina veterinaria. La Habana, Cuba: Ed CENSA. 103 p.

25. Sánchez A, Lamazares MC. 2010. Principales enfermedades que afectan a las aves. Tetramerosis. En: Sánchez A, López A, García MC, et al. (eds). Salud y producción de las aves. La Habana, Cuba: Ed Félix Varela. p 487-488.

26. Silva RJ, Oliveira-Sequeira TCG, Gurgel CC. 2005. Occurrence of Tetrameres confusa (Nematoda, Tetrameridae) in Ara ararauna (Psittacidae). Arq Bras Med Vet Zootec 57: 562-564. doi: 10.1590/S010209352005000400022

27. Todd FS. 1996. Natural history of the waterfowl. USA: San Diego Natural History Museum. 490 p.

28. Villarreal SM, Fedynich AM, Rollins D. 2011. Tetrameres pattersoni in bobwhites from the Rolling Plains Ecoregion of Texas. In: Fedynich AM (ed). Current Research 2010-2011. Texas, USA: Texas A\&M UniversityKingsville. p 52.

29. Zocche AT, Silva GS. 2009. Endoparasitoses em aves de produção industrial. $2^{\text {nd }}$ ed. In: Berchieri Junior A, Nepomuceno ES, Di Fabio J, et al. (eds). Doenças das aves. Brasil: FACTA. p 909-921. 